\title{
Effect of Phase 1 Cardiac Rehabilitation to Non-Invasive Hemodynamics in Acute Myocardial Infarction Patients
}

\author{
Desi Natalia Trijayanti Idris ${ }^{1}$, Dian Taviyanda ${ }^{1}$, Srinalesti Mahanani ${ }^{1}$ \\ ${ }^{1}$ Lecturer of Nursing Study Bachelor Program, STIKES RS Baptis Kediri
}

\begin{abstract}
Cardiovascular emergencies often occur suddenly, delays in treatment cause the condition to worsen. One of the cases of cardiovascular emergency is acute myocardial infarction (AMI). AMI patients must have stable hemodynamics in order to improve their condition. Management of nursing care that can be done to maintain hemodynamic stability of AMI patients is the implementation of Phase 1 Cardiac Rehabilitation. The purpose of this study was to analyze the effect of Phase 1 Cardiac Rehabilitation on Non-Invasive Hemodynamics for Acute Myocardial Infarction Patients in the ICCU Room of Kediri Baptis Hospital. The research design used a quasy experiment one group pre post test design. Population in this study were all AMI patients who were treated in the ICCU Room of the Kediri Baptist Hospital who were taken using a quota sampling technique with a sample size of 35 respondents. The instrument used was an observation sheet including: systolic - diastolic blood pressure and heart rate. Phase 1 cardiac rehabilitation program intervention was carried out for 2 consecutive days, with 2 training sessions/day (morning and evening). Each training session with a duration of 15 minutes. The data analysis of this study used the Paried T-Test statistical test. The results of this study showed that $p=0.026, p=0.014 p<0.05$ and that $p=0.024 p<0.05$, which means there is an effect of phase 1 cardiac rehabilitation on cytolic, diastolic blood pressure and heart rate in acute myocardial infarction patients in the ICCU room of Kediri Baptist Hospital. Conclusion Cardiac Rehabilitation Phase 1 has a significant effect on heart muscle function based on the results of blood pressure checks seen from systolic and diastolic and heart rate. Where the greatest significance is on heart rate compared to blood pressure.
\end{abstract}

Keywords: Cardiac Rehabilitation Phase 1, Non-Invasive Hemodynamics, Blood Pressure, Heart Rate.

\section{Introduction}

Acute Myocardial Infarction (hereinafter referred to as AMI) is myocardial necrosis caused by an imbalance between the supply and demand for oxygen to the heart muscle $^{1}$ and or AMI is a disease that attacks the heart organ. Which causes cell death in the heart muscle due to a long myocardial ischemic process in the heart ${ }^{2}$. Patients with AMI must receive therapy for at least 24 hours, and preferably for the index hospitalization duration, up to 8 days or until the patient does not feel the symptoms of infarction, namely no chest ${ }^{3}$. Myocardial ischemia will occur in the initial state if reperfusion action is not taken immediately, this can lead to permanent (irrevesible) heart necrosis. AMI can cause complications due to ventricular remodeling and ultimately can lead to cardiogenic shock, congestive heart failure, and persistent ventricular dysrhythmias. Management of action on AMI cannot be delayed if there is a delay every 30 minutes it can increase the risk of death by about $80 \%{ }^{4}$. During treatment it is necessary to observe to see whether the patient's hemodynamics have increased or decreased. It is hoped that the hemodynamic condition after an AMI attack can be maintained at normal levels/ conditions.

Based on data from the American Heart Association in 2014, heart disease is the cause of 1 in every 6 deaths in the United States in 2010 . Heart disease causes 7.2 million deaths per year worldwide ${ }^{6}$. AMI and its associated functional disorders can cause heart failure affecting $2-3 \%$ of the population in industrialized countries with an increase in age $>65 \mathrm{yr}$. A combined estimate of 20 million people have heart failure in Europe and the US and its prevalence is increasing. The highest Case Fatality Rate (CFR) occurred in AMI (13.49\%) and 